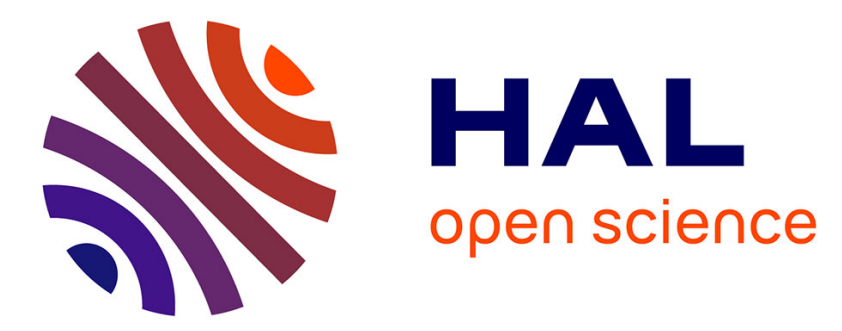

\title{
On Predation-Commensalism Processes as Models of Bi-stability and Constructive Role of Systemic Extinctions
}

\author{
E. Sanchez-Palencia, J.-P. Françoise
}

\section{- To cite this version:}

E. Sanchez-Palencia, J.-P. Françoise. On Predation-Commensalism Processes as Models of Bi-stability and Constructive Role of Systemic Extinctions. Acta Biotheoretica, inPress, 10.1007/s10441-02109413-3 . hal-03244151

\section{HAL Id: hal-03244151 https://hal.sorbonne-universite.fr/hal-03244151}

Submitted on 1 Jun 2021

HAL is a multi-disciplinary open access archive for the deposit and dissemination of scientific research documents, whether they are published or not. The documents may come from teaching and research institutions in France or abroad, or from public or private research centers.
L'archive ouverte pluridisciplinaire HAL, est destinée au dépôt et à la diffusion de documents scientifiques de niveau recherche, publiés ou non, émanant des établissements d'enseignement et de recherche français ou étrangers, des laboratoires publics ou privés. 


\title{
ON PREDATION - COMMENSALISM PROCESSES AS MODELS OF BI - STABILITY AND CONSTRUCTIVE ROLE OF SYSTEMIC EXTINCTIONS
}

\author{
E. SANCHEZ-PALENCIA, J-P FRANÇOISE
}

\begin{abstract}
We propose a mathematical model for a class of predator - prey systems more complex than the usual one, involving a commensalism effect consisting in an influence of the predator on the sustainability of the prey. This effect induces interesting new features, including bi-stability (two attractors with disjoint attraction basins). The question of the possibility of reaching a certain attractor starting from initial conditions with a small population of predators, which presents an interest from the vewpoint of the onset of the predator in evolution, is addressed. We propose two possibilities: the classical one involving adapted conditions in the far past and a new (up to our knowledge) one using biodiversity, specifically the presence of another predator which operates as a starter, being displaced in the sequel.
\end{abstract}

\section{INTRODUCTION}

This paper is devoted to a class of predator - prey systems with a functional structure more complex than the usual one, involving a supplementary commensalism effect in an influence of the predators on the subsistences of the preys. As an example of this ecological behaviour, we may think about a population of predators (insects) which are parasites of the preys (herbivores) but which in addition improve pollinization, so enhancing the equilibrium population of the preys. This new effect induces interesting new features, including bi-stability (simultaneous presence of two attractors with disjoint attraction basins). This rouses interesting questions, which present an interest from the viewpoint of evolution, concerning the possibility of reaching a certain attractor from initial data with a small population of predators.

1991 Mathematics Subject Classification. Primary 92D25 Population Dynamics, 92D40 Ecology, 37G15 Bifurcations of limit cycles and periodic orbits.

Key words and phrases. Predator-Prey Systems, Bi-stability, Excitability, "diversity - stability debate".

The authors are grateful to Philippe Lherminier for several enlightening discussions. 
As a matter of fact, the behavior of the predators is classical (their only resource are the preys) whereas the preys have their own subsistence. The preys are obviously consumed by the predators, but there is an extra positive effect of the predators on the subsistences of the preys. Consequently the action of the predator on the prey is two-fold, involving opposite effects of qualitatively different nature which cannot balance, so inducing new qualitative phenomena.

In our opinion, the very interest of the model is in exhibiting a variety of demographic phenomena and peculiarities of the stability effects induced by the complex action of the predators on the preys, rather than the specific knowledge of a certain type of such an action. But it is neverthelesss worthwhile thinking that such kind of behavior could be used for modelling situations where the predators are insects having a double action on the preys (herbivores), living at their expenses but also improving pollinization which turns out in enhancing the capacity of the susbtrate of the preys.

In situations where the efficiency of the predation is small, not allowing an invasive character of the preys, a sufficiently large effect of the predator on the substract of the preys induces the onset of an attractor with a (restricted) attraction basin disjoint of the axis of the preys (so unreachable by usual invasion of the predators). The problem of the invasion of such kind of attractors is not new in ecological dynamics, and it is usually solved by invoking different conditions in the far past. We adress this question in two ways. First, by constructing a (non-autonomous) system with variable parameters (so driven by external agents), and we give an example of such situation. But we also present a very different possibility, only involving autonomous systems (and then not needing external agents) which consists in the presence of another species of predators which operate as a starter allowing invasion of the predators, but displaced in the sequel by the first predator, which eventualy colonizes the attractor. This may be considered as a contribution to the so-called "diversity - stability debate" which include many related topics (see [9] and the references herein and $[11])$.

It is remarkable that the Predation-Commensalism system displays all the generic codimension-one bifurcations (saddle-node, AndronovPoincaré-Hopf and homoclinic bifurcations). This system has also the striking peculiarity to display excitability phenomenon, without being (in an obvious way) fast-slow. A key reference for bifurcation theory is [5]. See also [4] for bifurcation theory applied to biological oscillations. Excitability is usually well-known in dynamical systems applied 
to neurosciences (see for instance ([4], 4.8 p.92) and ([1], p.53) with FitzHugh-Nagumo and Morris-Lecar systems).

In this article, mathematics are practically reduced to description and comments on the basis of the numerical examples (done with Mathematica). We did not search to generalize the examples and we mainly focus on the description of certain mechanisms. Moreover, we do not search to give an explicit description of the admissible set of initial values, which is certainly not trivial; we only point out that all the reported facts are concerned with structurally stable properties, so that small perturbations of the parameters and of the initial values are allowed without changing the qualitative properties of the results.

The paper is organized as follows:

The Predation-Commensalism model is described in Section 2. Latter in the text, it will be refered to as the "PC-model".

In Section 3, we recall a few classical results of the model without Commensalism term in order to prepare the sequel.

In Section 4 we describe the main features of the Predation - Commensalism model.

Section 5 contains a further description of the homoclinic bifurcation.

Section 6 discusses the excitability phenomenon.

In Section 7 we give an explicit example of non-autonomous system allowing invasion of the predator and asymptotic approach to the nontrivial attractor.

Section 8 is devoted to a 3-dimensional system of two predators in competition for a prey, allowing also invasion of the non-trivial attractor.

The conclusions, dealing mainly with general items of evolution, are in Section 9.

\section{The Predation - Commensalism model}

The basic system is

$$
\left\{\begin{array}{l}
\dot{x}=a x(1-x / K)-y h(x) \\
\dot{y}=-c y+y h(x) .
\end{array}\right.
$$

where the functional response $h(x)$ is of the so-called "Holling II" type $([6],[7])$ i.e. proportional to $x$ for small $x$ with an asymptotic 
satiety bound. There are two significant parameters, the proportionality ratio $e$ (the efficiency of the predation with scarce preys) and $b$ (the satiety bound). Specifically, we shall take either

$$
h(x)=(b e x) /(b+e x)
$$

or

$$
h(x)=b \tanh (e x / b)
$$

$K$ is the total carrying capacity of the substrate of the preys $x$. Instead of the usual constant, we shall consider that $K$ depends either on the population of predators (4)

$$
K=K_{0}+\lambda y
$$

or on the predation activity $y h(x)$ itself (5)

$$
K=K_{0}+\lambda y h(x)
$$

The qualitative results are the same for (2) or (3) and for (4) or (5), and the choice for numerical computations will be specified in each example.

Obviously, the dynamics on the axis $x$ is not concerned with $\lambda$ and consists in a classical "logistic dynamics" with an unstable equilibrium at the origin and a stable one (attractor) at $x=K_{0}$. The invasive or non-invasive character of the predator $y$ relies on the behavior of $y^{\prime}$ in the immediate vicinity of this point. According to the second equation (1) invasion amounts to

$$
c<h\left(K_{0}\right)
$$

and is independent of $\lambda$. Using (for instance) the expression (3) we have

$$
(c / b)<\tanh \left(e K_{0} / b\right)
$$

In particular, it appears that, when fixing all the parameters unless $e$, invasion occurs for $e$ larger than a threshold given by the equation obtained from $(7)$ replacing $<$ by $=$. 


\section{BAsic Features of The PURE PREDATION System $\lambda=0$}

In this section we recall the main properties of system (1) with the functional response either (2) or (3) whith $K=K_{0}=$ const, i. e. $\lambda=0$ in (4) or (5).

The equilibrium $\left(x_{0}, y_{0}\right)$ is given by the solutions of the system (note that the second equation was divided by $y$ as we search for the internal equilibrium)

$$
\left\{\begin{array}{l}
0=a x_{0}\left(1-x_{0} / K_{0}\right)-y_{0} h\left(x_{0}\right) \\
0=-c+h\left(x_{0}\right)
\end{array}\right.
$$

Denoting by $h^{-1}$ the inverse function of $h$, which is well-defined on the interval $(0, b)$, we have

$$
\left\{\begin{array}{l}
\left.a x_{0}\left(1-x_{0} / K_{0}\right)\right)=c y_{0} \\
x_{0}=h^{-1}(c)
\end{array}\right.
$$

so that $x_{0}$ is given by the second equation and then $y_{0}$ by the first one. Fixing all the parameters unless the efficienty $e$ and using for instance (3), we have

$$
x_{0}=\frac{b}{e} \tanh (c / b),
$$

so that $x_{0}$ runs from $K_{0}$ to 0 for $e$ running from its lower value for invasion to $+\infty$. The equilibrium $\left(x_{0}, y_{0}\right)$ describes a parabola passing by $\left(K_{0}, 0\right)$ and the origin.

Several kinds of attractors then appear depending of the values of $e$ (see Fig 1; a more explicit description may be find in [13]). For $e$ less than the invasion threshold, the attractor is merely the equilibrium of the preys alone, $E$ (see Fig 1 with $e=0.15$ ). For larger values of $e$, there is an equilibrium $\left(x_{0}, y_{0}\right)$ on the parabola; it is stable (then an attractor, specifically a degenerate node), as in Fig 1 for $e=0.35$. When increasing $e$, the equilibrium becomes a stable focus (as in Fig $1 e=0.6$ ). With increasing values of $e$, (in particular on the left of the parabola) there is a Poincaré - Andronov - Hopf bifurcation and the equilibrium becomes unstable; it appears a periodic cycle around it which is the attractor (see Fig 1 with $e=0.85$ ). For even larger values of $e$, the cycle keeps dilating as $e \rightarrow+\infty$ and tends to become a curvilinear triangle passing nearby the origin and the equilibrium of the preys alone (see Fig 1 with $e=2$ ).

It appears that the above described pattern is concerned with values of $e$ sufficiently large to have invasion of the predators; for lower values 


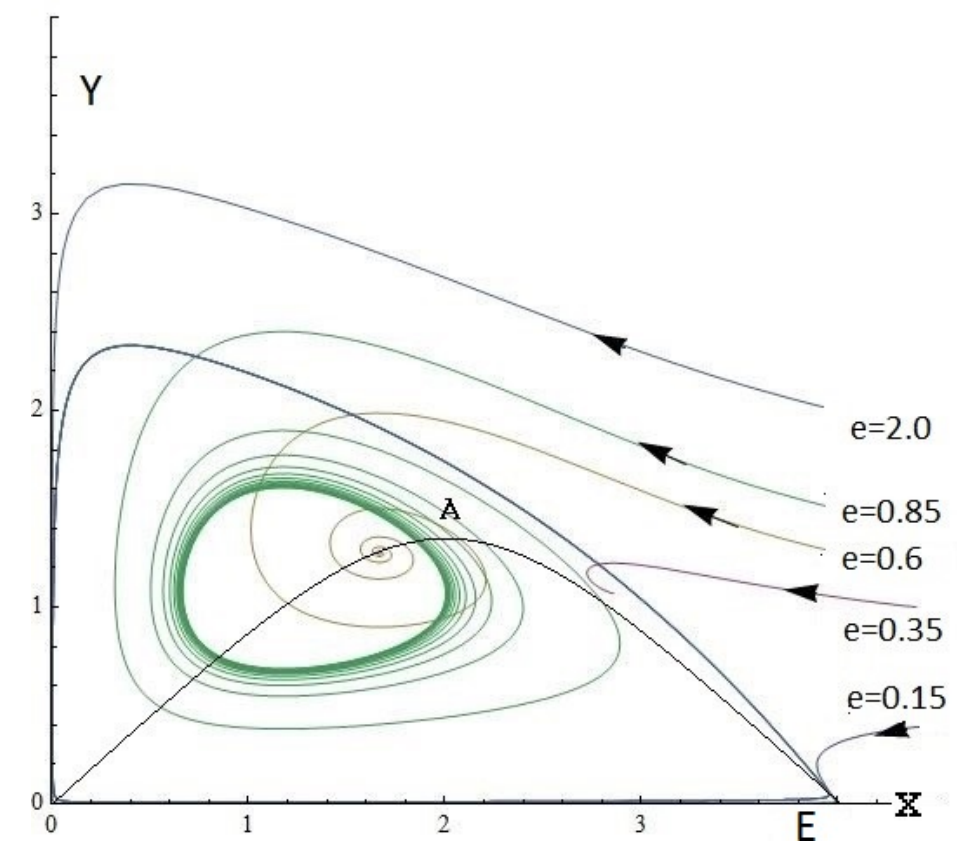

Figure 1. Plot of several orbits of system (1) with (3) for $a=b=1, c=0.76, K_{0}=4$ and several values of $e$ with the parabola $c y=x\left(1-\frac{x}{4}\right)$.

of $e$ the attractor is merely the point $\left(0, K_{0}\right)$ (equilibrium of the preys alone (with their subsistences)).

\section{Features of the Predation - Commensalism system$$
(\lambda>0)
$$

The results for the classical model $\lambda=0$ were explicitly described in the previous section. It was concerned with $e$ sufficiently large to have invasion of the predators from the vicinity of the equilibrium of the preys alone, $\left(K_{0}, 0\right)$. In this case, passing to $\lambda>0$ modifies the pattern so that the cycles are larger and often contain a part of them in the region $x>K_{0}$, but the main features are analogous. We only consider in the sequel the case of $e$ small, so that there is no invasion of the predators from the vicinity of $\left(K_{0}, 0\right)$.

In order to understand the role of parameter $\lambda$, let us fix in (1), (3) the parameters $a=0.5, b=1, e=0.6, K_{0}=1, c=0.8$, and let $\lambda$ run from 0 to $+\infty$.

For $\lambda=0$ we obviously have the classical system with non-invasive $y$, so that the attractor is merely the equilibrium of the preys alone, 


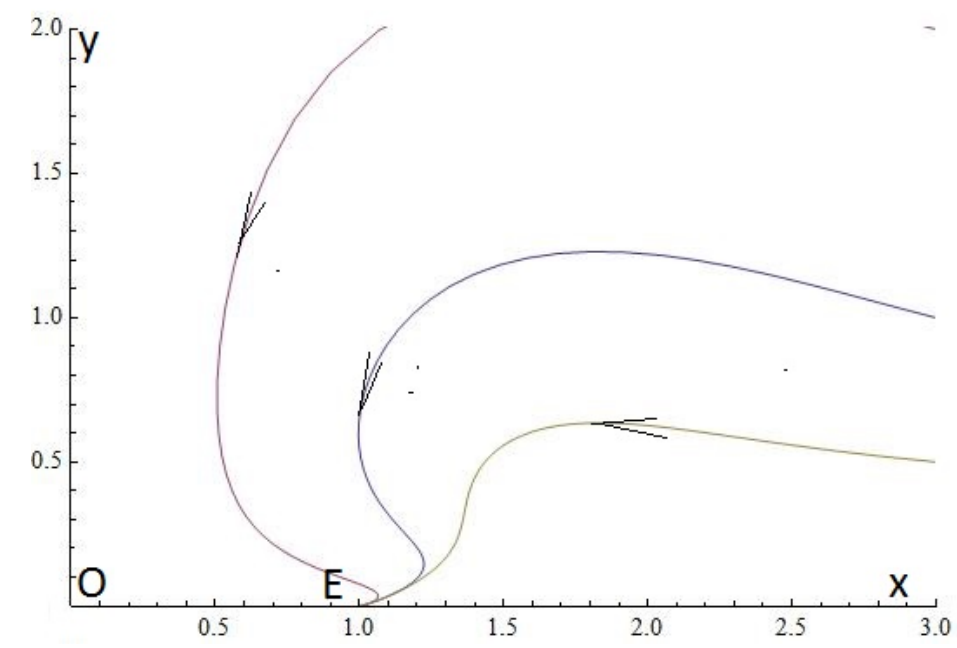

Figure 2. Phase portrait of (1), (3) with small $e=0.6$ and moderate $\lambda=3$ (see other parameters in the text). The attractor is the equilibrium of the preys alone, $E$.

$\left(K_{0}, 0\right)$. This pattern remains qualitatively the same for small $\lambda$ (see Fig 2 for $\lambda=3$ ):

With increasing $\lambda$, there is a saddle - node bifurcation with sudden inception of two equilibria (with the same $x_{0}$ ). With slightly larger $\lambda$ the node becomes an attractive focus. The phase portrait is seen in Fig 3 for $\lambda=5$. There is a bi-stability: the saddle $S$ has a stable manifold which is the common boundary of two attraction basins which send the positive half-orbits towards the focus $A$ and the equilibrium of the preys alone $E$ respectively. We note that the predator $y$ is always noninvasive from the vicinity of $E$, which is an attractor; the new attractor $A$ is only accessible from its attraction basin (the domain around $A$ enclosed by the stable manifold of $S$ ), which implies moderately large initial values of $y$.

Moreover, when increasing $\lambda$, there is a Poincaré - Andronov - Hopf bifurcation of the upper equilibrium, which loses its stability, and it appears a stable cycle around it. The phase portrait is seen in Fig 4 for $\lambda=6$. The upper equilibrium is an unstable focus (denoted $I$ ), and its attraction basin is analogous to the previous one (with the exception of the point $I$ itself, which is an unstable equilibrium).

For larger values of $\lambda$ the stable cycle $C$ dilates untill it touches the saddle $S$; there is a homoclinic bifurcation (which is more explicitly described in the next section). Increasing $\lambda$ there is again only one attractor, the equilibrium of the preys alone, but the phase portrait is 


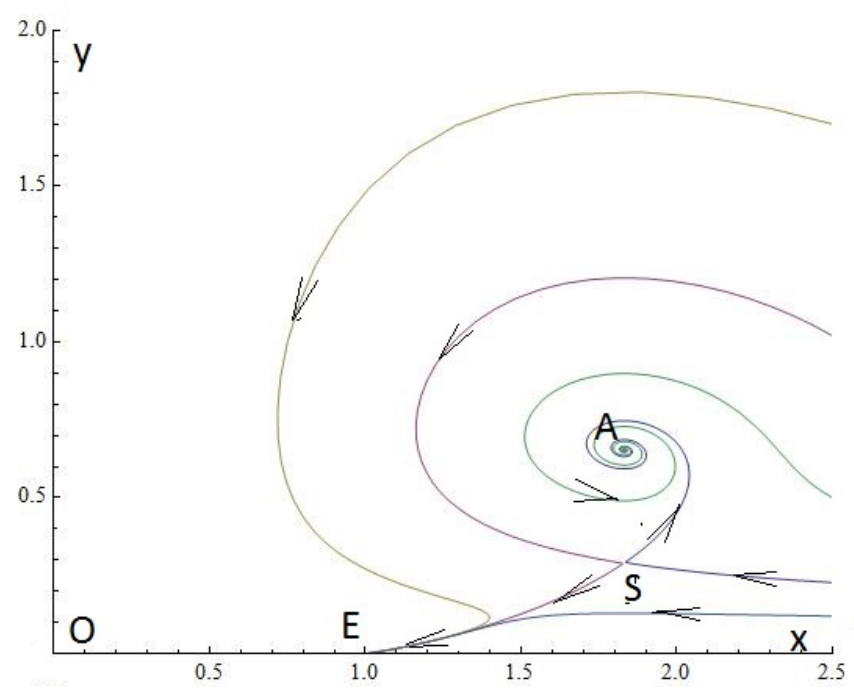

Figure 3. The phase portrait for $\lambda=5$ (other parameters are the same). There is a new attractor $A$ in addition to $E$. The stable manifold of the saddle $S$ separates the attraction basins of $A$ and $E$.

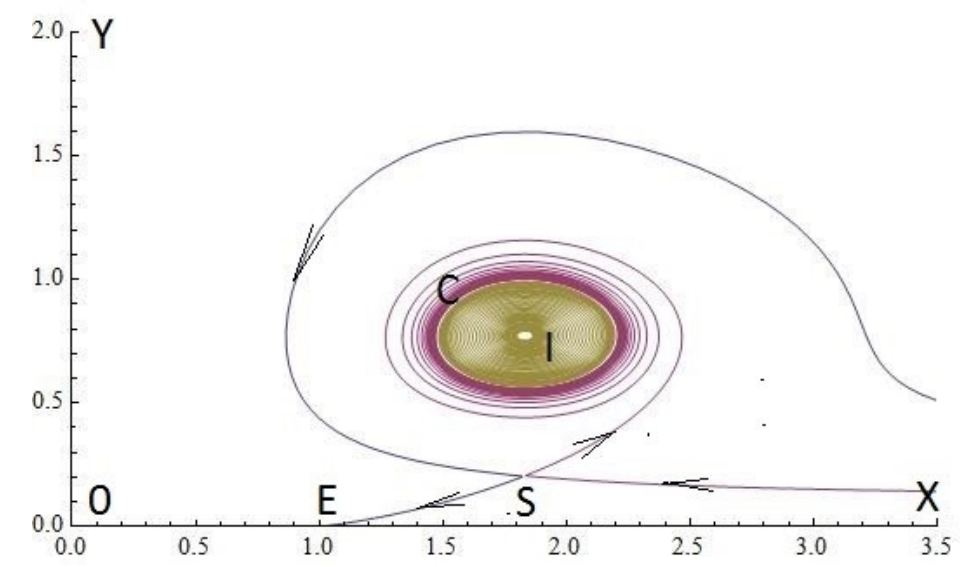

FiguRE 4. The phase portrait for $\lambda=6$ (other parameters are the same). The upper attractor is now the periodic cycle $C$ around the upper (unstable) equilibrium $I$. The stable manifold of the saddle $S$ separates the attraction basins of $C$ and $E$.

very different from that of Fig 2. It is shown for $\lambda=10$ in Fig 5 . The two branches (upper and down) of the unstable manifold of the saddle $S$ join $S$ to $E$, the down directly, and the upper after a large loop. 


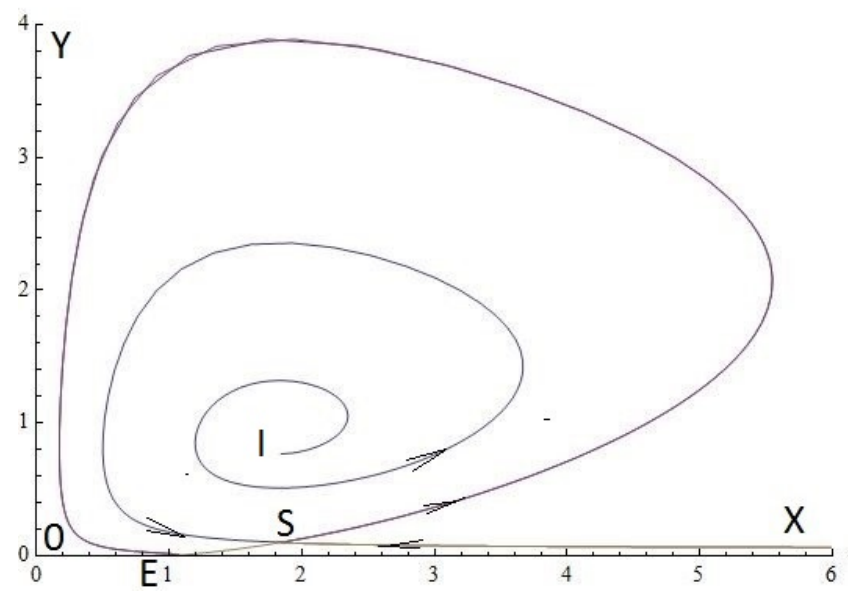

Figure 5. The phase portrait for $\lambda=10$, other parameters are the same. The upper half of the unstable manifold of $S$ sends to $E$, which is a global unique attractor (the bi-stability phenomenon disappeared). This global attractor displays a property of excitability.

The former attraction basin of the limit cycle is no longer trapped, it turns and eventually dumps in $E$, so that the bi-stability disappeared. This global attractor displays a property of excitability (which is more explicitly described in another section).

\section{Complements on the homoclinic BifurCAtion}

We give a few complements on the (somewhat unusual in two-dimensional predator-prey system) homoclinic bifurcation which intervenes between the patterns of Fig 4 and Fig 5 of the previous section. In order to have a clearer figure, we change a little the values of the parameters, which are now $a=0.4, b=1, e=0.5, K_{0}=1, c=0.716$. When increasing $\lambda$, the periodic cycle $C$ dilates, and, at $\lambda=11.18$ (Fig 6) it touches the saddle $S$. This homoclinic bifurcation has a non-local character, and it amounts to the fact that the upper half of the unstable manifold of $S$ becomes itself the cycle, it turns and comes back to $S$ again as the left branch of the stable manifold (it is a homoclinic orbit). It shoud be noted that the orbit itself starts and finishes in a classical equilibrium, so that the total time is infinite, in which it differs of a classical periodic cycle. Otherwise, it is clear that the points inside the cycle move towards it, whereas the outer points go to $E$. 


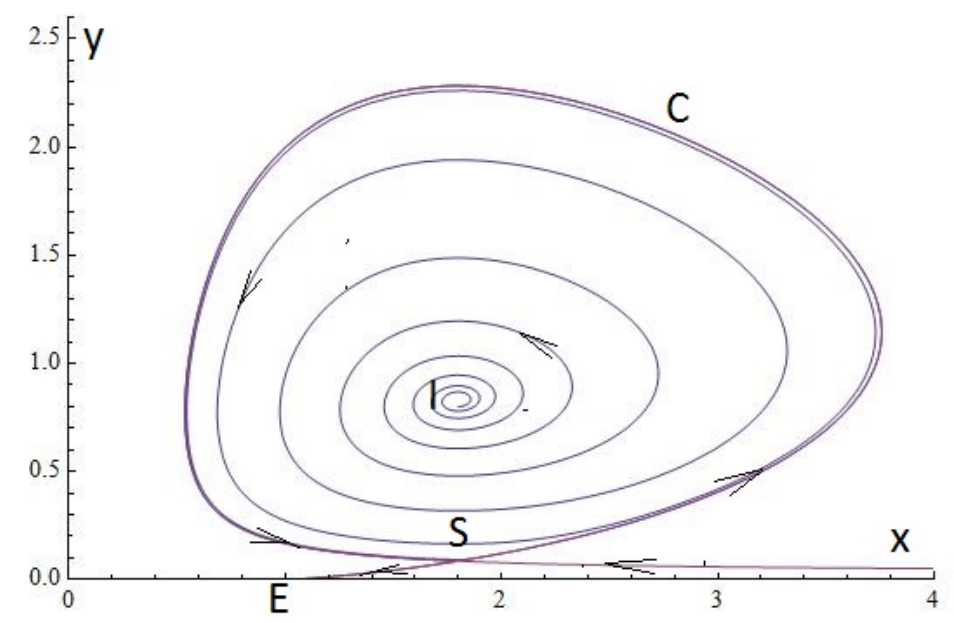

FiguRE 6. The homoclinic orbit corresponding to the (homocline) bifurcation for system (1), (3) with $a=0.4$, $b=1, e=0.5, K_{0}=1, c=0.716 \lambda=11.18$.

\section{THE PROPERTY OF EXCITABILITY}

At this point, it is worthwhile mentioning an interesting phenomenon of excitability which appears for $\lambda$ sligthly larger than the homocline bifurcation value. In that case, the phase portrait is almost the same as for the bifurcation (Fig 6), but the exact topology is that of Fig 5, so that the interior of the cycle eventually goes out and dumps into the equilibrium of the preys alone, $E$. As a matter of fact, starting inside the cycle, the orbit turns dilating and approaches the cycle, but ultimately it goes out towards $E$. Fig 7 is a plot of such solutions as function of time for $\lambda=11.19$ (the very bifurcation is at $\lambda=11.18 \ldots$ ). There is a similar global behaviour of the orbits with solutions of fastslow systems well-known as models of neurosciences like the FitzHughNagumo and the Morris-Lecar systems. More precisely if we plot the solution $y(t)$ with initial data $\left(x_{0}, y_{0}\right)$ there is a threshold $M\left(x_{0}\right)$ such that if the initial data $y_{0}<M$ then the solution decreases rapidly to its asymptotics; in contrast the solution $y(t)$ with initial data $y_{0}>M$ displays a large excursion in the phase portrait before decreasing to its asymptotics. Using the language of neurosciences, we say that the neuron presents an action potential before relaxing to its equilibrium. By analogy, we show in the next figure two orbits with one (respectively two) action potentials. This is rather surprising to see such a phenomenon in the class of prey-predator systems which furthermore looks a priori not fast-slow in an obvious way. 


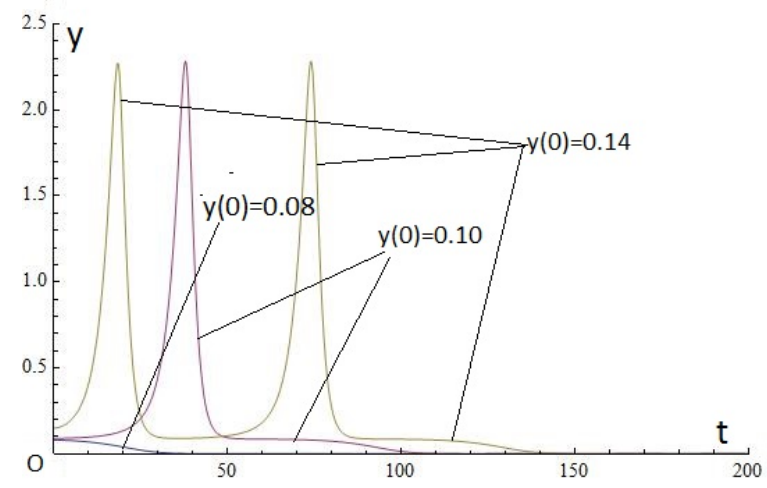

Figure 7. Plot of a solution of the same system of Fig 6 with a slightly larger $\lambda=11.19$ exhibiting excitability. If starting inside the cycle, it grows towards the cycle and eventually it goes out, to the equilibrium of the preys alone $E$.

\section{Attending the NON-TRIVIAL ATtraCtor IN CASE OF SLOWLY VARYING PARAMETERS}

We now focus on the attractors in the bi-stability case for intermediate values of $\lambda$ (either points as in Fig 3 or cycles as in Fig 4). It is clear that they cannot be reached in the previous framework starting with a small number of predators nearby the equilibrium of the preys alone, $E=\left(K_{0}, 0\right)$. The classical explanation of the possibility of such a fact is that the invasion of the predators $y$ occurred in a far past of evolution, when values of the parameters were others, allowing invasion; later, slowly, the values of the parameters changed, stabilizing at the present values, which only operate on "somewhat large" values of $y$, which lead to the attractor. We give now an example of such a system.

We consider the system

$$
\left\{\begin{array}{l}
\dot{x}=a x(1-x / K)-y b \tanh (e x / b)) \\
\dot{y}=-c y+y \tanh (e x / b)
\end{array}\right.
$$

with the parameters $c, e$ and $\lambda$ are functions of time, so that the system is non-autonomous. Moreover, as $\mu$ is small, the parameters are slowly variable: 


$$
\left\{\begin{array}{l}
a=0.5, \quad b=1, \quad \mu=0.01 \\
K=1+\lambda y \\
\lambda=5-e^{-\mu t} \\
c=0.8-0.3 e^{-\mu t} \\
e=0.6+1.2 e^{-\mu t} .
\end{array}\right.
$$

We first note that these parameters converge asymptotically to constant values corresponding to a bi-stability with a point attractor, as in Fig 3. Moreover, the values of the parameters for $t=0$ (in particular with a larger value of $e$, when taken constant, give an autonomous system with invading properties for $y$, leading to a cyclic attractor (as in Fig 1 for $e=0.85$ for instance).

Fig 8 is a superposition of three orbits. The first one corresponds to the autonomous system with the (constant) asymptotic values (12) for large $t$ and starts from any point of the attraction basin of the upper equilibrium. The second one is concerned with the constant values of parameters (12) for $t=0$, and starts from the very vicinity of the equilibrium of the preys alone, $E$; it obviously converges to the cyclic attractor on the left of Fig 8. The third one is the very orbit corresponding to the non-autonomous system with the variable parameters (12), starting, as the second, from the vicinity of $E$. Initially it is somewhat analogous to the second orbit, but afterwards it evolves approaching gradually of the asymptotic limit, which is the point attractor of the first orbit.

\section{TWO PREDATORS IN COMPETITION FOR A PREY AND "STARTER" PHENOMENON}

There is a huge development of mathematical studies of 3-dimensional systems associated with two predators in competition for a prey ([10, $3,2,14,15])$, in particular with the discussions of "competitive exclusion", persistence of diversity and invasion [12]. See also [8] for the existence of relaxation oscillations in this context.

We present another possibility for attending the attractor in the case of bi-stability from the vicinity of the equilibrium of the preys alone $E$, only involving autonomous systems, i. e. constant parameters. The idea consists in introducing another predator $y_{2}$ with parameters different from those of the previous $y$, now denoted by $y_{1}$. The parameters are chosen in such a way that $y_{2}$ alone with $x$ is invasive (in particular the efficiency $e_{2}$ is larger than $e_{1}$ ), so allowing invasion of $y_{2}$ (with a small quantity of $y_{1}$ ) but such that, when the populations are large, $y_{1}$ displaces $y_{2}$ (in particular the ratios of death are $c_{2}>c_{1}$ ), so that the 


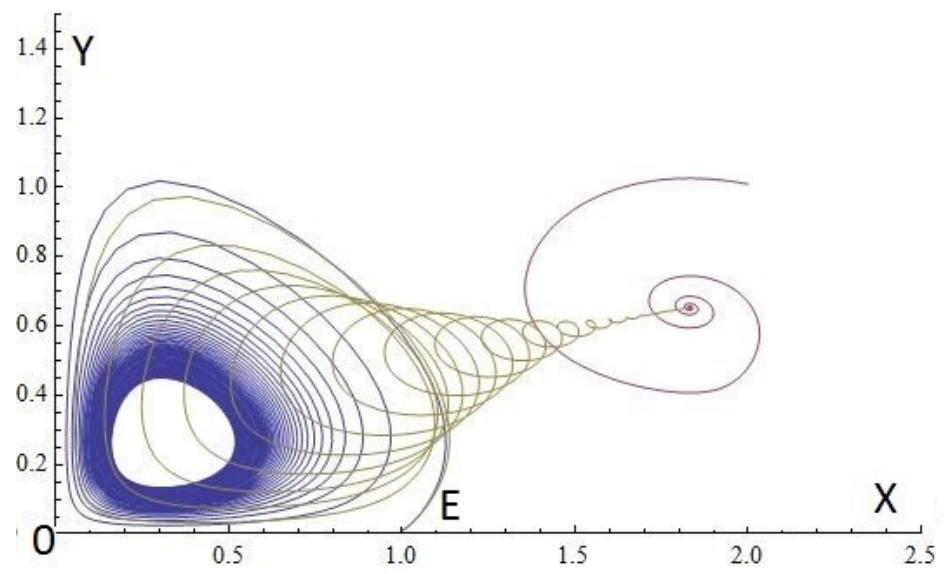

FiguRE 8. Plot of an orbit of the non-autonomous system (11), (12), starting from the vicinity of $E$. Initially it is somewhat analogous to the solution for the autonomous system with the initial values of the parameters, but afterwards it evolves approaching gradually of the asymptotic limit, corresponding to the final values of the parameters. Two orbits for the autonomous systems corresponding to the initial and final values of the parameters are also plotted.

attractor is on the plane $y_{2}=0$. The auxiliary predator $y_{2}$ plays the role of a "starter".

Specifically, let us consider the system (13)

$$
\left\{\begin{array}{l}
\left.\dot{x}=a x(1-x / K)-y_{1} b_{1} \tanh \left(e_{1} x / b_{1}\right)\right)-y_{2} b_{2} \tanh \left(e_{2} x / b_{2}\right) \\
\left.\dot{y_{1}}=-c_{1} y_{1}+y_{1} b_{1} \tanh \left(e_{1} x / b_{1}\right)\right) \\
\left.\dot{y_{2}}=-c_{2} y_{2}+y_{2} b_{2} \tanh \left(e_{2} x / b_{2}\right)\right)
\end{array}\right.
$$

with the (constant) parameters (14)

$$
\left\{\begin{array}{l}
a=0.5, \quad b_{1}=1.7, \quad b_{2}=1 \\
\left.\left.K=1+\lambda_{1} y_{1} b_{1} \tanh \left(e_{1} x / b_{1}\right)\right)+\lambda_{2} y_{2} b_{2} \tanh \left(e_{2} x / b_{2}\right)\right) \\
e_{1}=0.72, \quad e_{2}=1.33 \\
\lambda_{1}=10, \quad \lambda_{2}=6 \\
c_{1}=0.72, \quad c_{2}=0.86
\end{array}\right.
$$

There are orbits starting from $x(0) \approx 1$, small $y_{2}(0)$ and very small $y_{1}(0)$ (specifically, starting from $(1,0.001,0.02)$ ) which tend asymptotically to the attractor on $y_{2}=0$. Indeed, Fig 9 is a plot of this orbit 


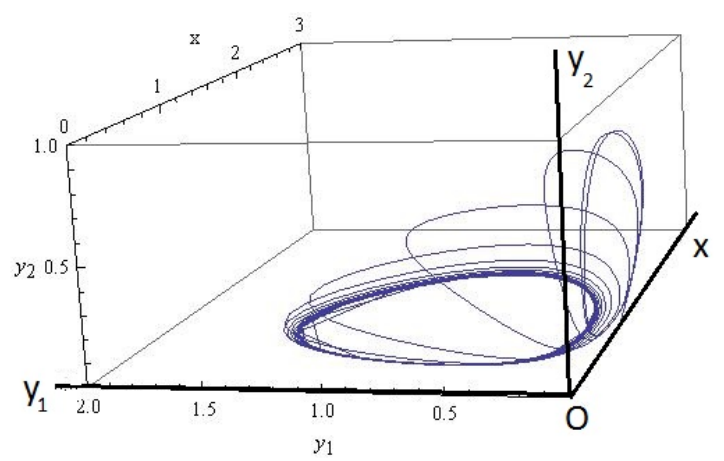

Figure 9. Plot of a three-dimensional orbit of the autonomous system (13), (14) starting nearby the equilibrium $E$ of the preys alone and tending to the cyclic attractor on the plane $y_{2}=0$.

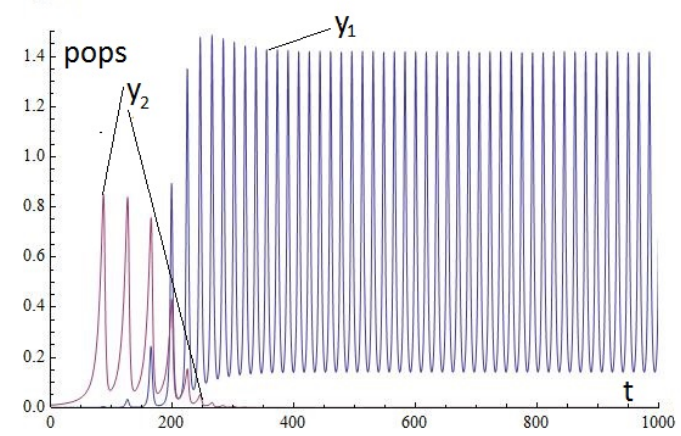

Figure 10. Plot of $y_{1}(t)$ and $y_{2}(t)$ of the previous figure 9 . The predator $y_{2}$ is initially essential and dies out, whereas $y_{1}$ is initially very small but attends the attractor on the plane $x_{2}=0$

(it starts nearby the axis $x$, it spirals in the three-dimensional space and it tends to a point attractor $A$ on the plane $\left.y_{2}=0\right)$. The populations of the two predators as functions of $t$ are shown in Fig 10, where it is apparent the booster role of $y_{2}$, which is initially essential, but disappears asymptotically for large $t$.

\section{Conclusion}

There are two essential points in this work. The first one is the wide range of phenomena opened by the twofold (demographic) action of the predators on the preys. In addition to the obvious consumption of preys, the predators have a positive influence on the subsistences of the preys, which constitutes a demographic advantage. These two actions 
have clearly positive and negative influences on the preys, but they are qualitatively different, so non-comparable, inducing new qualitative phenomena, including bi-stability and excitability.

The second is a (new up to our knowledge) setting in ecological dynamics allowing "jumps" from an attraction basin to another in problems with bi-stability with constant parameters (autonomous systems), different of the usually evoked slow variation of the parameters in the far past (non-autonomous systems) inducing a re-arrangement of the topology of the attraction basins. This new setting consists in the presence of another species operating as a starter which disappears asymptotically in the sequel, but allowing a drastic modification of the topology as the dimension of the phase space is improved. It then appears that the keypoint of this setting is the biological diversity (two predators with different parameters) instead of the externaly driven change of the parameters usually evoked.

These two items should then be considered as workable tools of the evolution with an intrinsic (epistemological) interest independent of the specific problems handled here. 


\section{REFERENCES}

[1] Ermentrout, G. Bard; Terman, David H., Mathematical foundations of neuroscience. Interdisciplinary Applied Mathematics, 35. Springer, New York, 2010.

[2] G.J. Butler, P. Waltman Bifurcation from a limit cycle in a two predator-one prey ecosystem modeled on a chemostat J. Math. Biol., 12 (1981), pp. 295-449.

[3] S.-B. Hsu, S.P. Hubbell, P. Waltman Competing predators. SIAM J. Appl. Math., 35 (1978), pp. 617-625.

[4] J.-P. Françoise Oscillations en biologie. (French) [Oscillations in biology] Analyse qualitative et modèles. [Qualitative analysis and models] Mathématiques Applications (Berlin) [Mathematics Applications], 46. Springer-Verlag, Berlin, 2005

[5] Shui-Nee Chow, Chengzhi Li, Duo Wang, Normal Forms and Bifurcation of planar vector fields. Cambridge University Press (Cambridge) 1994.

[6] C.S. Holling Some Characteristics of Simple Types of Predation and Parasitism The Canadian Entomologist vol. 91,, p. 385-398. doi:10.4039/Ent91385-7. ( 1959).

[7] C. S. Holling The components of predation as revealed by a study of small mammal predation of the European Pine Sawfly The Canadian Entomologist, vol. 91, p. 293-320 (1959).

[8] Liu, Weishi; Xiao, Dongmei; Yi, Yingfei Relaxation oscillations in a class of predator-prey systems. J. Differential Equations 188 (2003), no. 1, 306331.

[9] K. S. McCann The diversity - stability debate Nature 405 p. 228-230 ( 11 may 2000).

[10] R. McGehee, R.A. Armstrong Some mathematical problems concerning the principle of competitive exclusion J. Differential Equations, 23 (1977), pp. 3052.

[11] D. Melchionda, E. Pastacaldi, C. Perri, M. Banerjee, E. Venturino Social behavior-induced multistability in minimal competitive ecosystems J. Theoretical Biology, 439 (2018), pp. 24-38

[12] E. Sanchez-Palencia, J.-P. Françoise, New Schemes of Dynamic Preservation of Diversity: Remarks on Stability and Topology. Acta Biotheor 68, 157169 (2020).

[13] E. Sanchez-Palencia, Ph. Lherminier, Paradoxes of vulnerability to predation in biological dynamics and mediate versus immediate causality Disc. Cont. Dynam. Systems Series S, 13(8) p. 2195 - 2209,(2020).

[14] H.L. Smith Competitive coexistence in an oscillating chemostat SIAM J. Appl. Math., 40 (1981), pp. 498-522.

[15] H.L. Smith The interaction of steady state and Hopf bifurcation in a twopredator-one-prey competition model SIAM J. Appl. Math., 42 (1982), pp. 2743.

Sorbonne Université, UPMC Univ Paris 06, And CNRS, UMR 7190, Institut Jean Le Rond D'Alembert, F-75005, Paris France, Sorbonne Université, UPMC Univ PARIS 06, and CNRS, UMR 7598, Laboratoire JaCQUeS-LOUis LiOns, F-75005, PARis France

E-mail address: sanchez@dalembert.upmc.fr, Jean-Pierre.Francoise@Sorbonne-Universite.fr 